\title{
Femtosecond laser processing of glassy and polymeric matrices containing metals and semiconductor nanostructures
}

\author{
J.M.P. Almeida ${ }^{a}$, V. Tribuzi ${ }^{\text {}}$, R.D. Fonseca ${ }^{a}$, A.J.G. Otuka ${ }^{a}$, P.H.D. Ferreira ${ }^{\text {a }}$, V.R. Mastelaro ${ }^{\text {a }}$, P. Brajato ${ }^{\text {a }}$, \\ A.C. Hernandes ${ }^{a}$, A. Dev ${ }^{a, d}$, T. Voss ${ }^{b}$, D.S. Correa ${ }^{c}$, C.R. Mendonca ${ }^{a, *}$ \\ a Instituto de Física de São Carlos, Universidade de São Paulo, 13560-970, São Carlos, SP, Brazil \\ ${ }^{\mathrm{b}}$ Institute of Solid State Physics, University of Bremen, 28359 Bremen, Germany \\ ' Laboratório Nacional de Nanotecnologia para o Agronegócio (LNNA), Embrapa Instrumentação, 13560-970, São Carlos, SP, Brazil \\ ${ }^{\mathrm{d}}$ School of Information and Communication Technology, Royal Institute of Technology (KTH), Electrum 229, S-16440 Kista, Sweden
}

\section{A R T I C L E I N F O}

\section{Article history:}

Received 8 January 2013

Received in revised form 25 July 2013

Accepted 1 August 2013

Available online 26 August 2013

\section{Keywords:}

Femtosecond laser

Material processing

Two-photon polymerization

Metallic nanoparticles

Hybrid nanomaterials

\begin{abstract}
A B S T R A C T
Tailoring properties of materials by femtosecond laser processing has been proposed in the last decade as a powerful approach for technological applications, ranging from optics to biology. Although most of the research output in this field is related to femtosecond laser processing of single either organic or inorganic materials, more recently a similar approach has been proposed to develop advanced hybrid nanomaterials. Here, we report results on the use of femtosecond lasers to process hybrid nanomaterials, composed of polymeric and glassy matrices containing metal or semiconductor nanostructures. We present results on the use of femtosecond pulses to induce $\mathrm{Cu}$ and $\mathrm{Ag}$ nanoparticles in the bulk of borate and borosilicate glasses, which can be applied for a new generation of waveguides. We also report on 3D polymeric structures, fabricated by two-photon polymerization, containing $\mathrm{Au}$ and $\mathrm{ZnO}$ nanostructures, with intense two-photon fluorescent properties. The approach based on femtosecond laser processing to fabricate hybrid materials containing metal or semiconductor nanostructures is promising to be exploited for optical sensors and photonics devices.
\end{abstract}

(c) 2013 Elsevier B.V. All rights reserved.

\section{Introduction}

The need for developing advanced hybrid materials based on polymers, glasses, and ceramics with enhanced properties has increased dramatically in the last few years. Further development for processing such materials in a fast, effective, and low-cost way, however, is still on demand. Among the technologies up-todate employed for processing hybrid advanced materials, femtosecond laser processing (FLP) [1-5] stands out due to its high precision, low heat generation, and capability to design 2D and 3D structures, providing the materials with new architectures and properties.

The fabrication tailoring of these hybrid materials by FLP depends mainly on the laser intensity. Thus, the use of ultra-short laser pulses (50-150 fs), with pulse energy ranging from $\mathrm{nJ}$ to $\mathrm{mJ}$, as well as, tight focusing lens with high NA are required. As FLP involves nonlinear absorption, it is desirable that the material does not absorb the wavelength of the laser. However, it is possible to micromachining the bulk of materials that have electronic transitions close to the energy of the incident photon, in which multi-

\footnotetext{
* Corresponding author. Tel.: +55 1633738085.

E-mail address: crmendon@ifsc.usp.br (C.R. Mendonca).
}

photon absorption, confined in the focal volume, is responsible for the microstructuring. Ordinary oscillation of the laser beam does not affect the fabrication due to fast processing.

One interesting application for femtosecond laser processing (FLP) regards the fabrication of three-dimensional polymeric micro/nanostructures using two-photon polymerization (2PP) [6,7]. 2PP takes place when two photons are simultaneously absorbed by the chemical species responsible for triggering the polymerization, which consequently hardens the polymeric resin in a well confined spatial region. Considering that 2PP shows a quadratic dependence on the light intensity, as a consequence of the twophoton absorption nature, such process yields high spatial resolution and low light scattering. Another important feature for 2PP is related to the polymerization threshold, which directly influences the generation of radical species. By controlling the laser intensity at the focal volume, one can create radicals by two-photon absorption (2PA), that are neither suppressed by oxygen (which would reduce or hinder voxel growth), nor polymerize large voxels which are not desired for micro/nanofabrication. Therefore, structures with dimensions below the diffraction limit can be obtained [68]. For instance, two-photon polymerization has been employed in micro/nanofabrication for applications in optics, biology and microfluidics [3,6,9-14]. 
Femtosecond laser has also been used for processing glassy materials in order to fabricate two- and three-dimensional structures, including optical components, such as waveguides and resonators $[1,15,16]$. For microstructuring glasses, one usually takes advantage of FLP to induce nonlinear optical interactions, such as multi-photon absorption or ionization. Such processes involve plasma generation and its subsequent absorption of the laser energy causes irreversible damages to the material [17]. In glasses, such damages can result in a change of the refractive index, absorption coefficient, nonlinear optical susceptibility, structure and local composition [18]. Changes in the valence states of some ions have also been observed, which is useful for the generation of localized nanoparticles in glass with distinct shapes $[19,20]$. As an example of these applications, the formation of copper nanoparticles in a borosilicate glass by fs-laser micromachining has been demonstrated [21].

Metallic nanoparticles have attracted great attention because of their unique optical and electronic properties. Their use in nanoplasmonics has been proposed for applications in cancer treatments [22,23], chemical and biological sensing [24,25] and photovoltaic cells [26]. By using such nanoparticles it is possible to tailor the optical properties of micro devices, for example through the local field enhancement effect. Furthermore, the characteristic plasmon absorption band is sensitive to the surrounding medium making microdevices doped with nanoparticles suitable for sensor applications. In the same way, semiconductor nanostructures have also been widely exploited for technological applications, in which $\mathrm{ZnO}$ nanowires have a fundamental role. Due to its wide-bandgap $(3.37 \mathrm{eV}$ ) and large exciton binding energy $(60 \mathrm{meV}), \mathrm{ZnO}$ has been used for the development of UV photodetectors, optical switches and nonlinear frequency converters. Moreover, $\mathrm{ZnO}$ nanowires can be used in hybrid structures along with polymeric matrices for fabrication of efficient UV or white LEDs [27].

On an account of quantum confinement effects, many studies have been directed to the incorporation of $\mathrm{Au}, \mathrm{Ag}$ or $\mathrm{Cu}$ nanoparticles and Zno nanowires in different matrices, resulting in a nanocomposite or hybrid material. Although considerable advances have been achieved for the production of macroscopic samples, the preparation of these hybrid materials in the microscopic scale are still on demand.

In this work we report on the use of femtosecond laser for processing new nanocomposites in the micrometer scale, including (i) polymer-based structures, fabricated by $2 \mathrm{PP}$, containing gold or $\mathrm{ZnO}$ nanowires, with luminescence properties that can be tuned according to the excitation wavelength and (ii) borate and borosilicate glassy matrices doped with silver and cooper, where metal nanoparticles are produced by the laser irradiation, with interesting optical properties.

\section{Experimental}

The microfabrication techniques employed in this work, twophoton polymerization (2PP) and fs-laser micromachining, are schematically presented in Fig. 1(a and b), respectively.

For the 2PP, illustrated in Fig. 1(a), two scanning mirrors and a motorized stage are used to scan the laser beam through the sample. The motorized stage moves (50 $\mathrm{nm}$ minimal incremental motion) the sample along the beam propagation direction $(z)$, while a pair of galvanometric mirrors deflects the beam in the $x$ and $y$ directions, allowing for the fabrication of three-dimensional structures. The laser beam is focused into the sample using a microscope objective. A red light emitting diode (illumination at $600-680 \mathrm{~nm})$ and a $(640 \times 480)$ CCD camera are used for monitoring the fabrication in real time. We used a Ti: Sapphire laser, operating at $82 \mathrm{MHz}$ that delivers $\sim 50 \mathrm{fs}$ pulses at $790 \mathrm{~nm}$ ( $40 \mathrm{~nm}$ bandwidth), as the excitation source for the 2PP. In the vicinity of the focal volume, the light intensity is high enough, such that the photoinitiator undergoes two-photon absorption, locally promoting the sample polymerization. After the microfabrication, the samples are immersed in ethanol to wash away all uncured resin, leaving only the fabricated microstructures adhered to the substrate.

The base resin used in the 2PP microfabrication is composed of two triacrylate monomers; tris(2-hydroxyethyl) isocyanurate triacrylate which gives hardness to the microstructure and ethoxylated(6) trimethylolpropane triacrylate which is responsible for reducing shrinkage upon polymerization. The ratio between each monomer in the resin can be chosen to tailor the final polymer mechanical properties [28]. The monomers are mixed in ethanol and then the liquid photoinitiator ethyl-2,4,6-trimethylbenzoyl phenylphosphinate is added in 3 weight\% in excess [29].

The method developed for indirectly doping the microstructures with gold nanoparticles is carried out in two steps according to [30]. The first step consists of mixing the monomers with an aqueous solution of $\mathrm{HAuCl}_{4}(2 \mathrm{~g} / \mathrm{l})$. The components were mixed in a proportion of $1 \mathrm{ml}$ of the solution to $2.5 \mathrm{~g}$ of the resin for $1 \mathrm{~h}$. After mixing, the sample is left for $24 \mathrm{~h}$ in a $50{ }^{\circ} \mathrm{C}$ chamber for evaporation of the solvents and then it is ready for the 2PP fabrication. The second step takes place after the microstructure fabrication and washing process. The sample is then heated to $185^{\circ} \mathrm{C}$ for $35 \mathrm{~min}$, which promotes the reduction of gold ions to metallic gold, with the polymer acting as the reducing agent. Also, by raising the temperature we increase the mobility of the atomic species inside the microstructure, promoting the formation of gold nanoparticles.

ZnO nanowires were prepared by a hydrothermal technique from an aqueous solution of potassium hydroxide and zinc nitrate. A glass beaker containing a homogeneous and transparent solution of the reactants was placed on a hot plate at $80^{\circ} \mathrm{C}$ for about $4 \mathrm{~h}$. After the reaction a milky white precipitation of $\mathrm{ZnO}$ nanowires was collected from the beaker and washed several times with water and ethanol. The obtained nanowires are $1-3 \mu \mathrm{m}$ long and with diameters of approximately $100 \mathrm{~nm}$. After the monomers are mixed to the phoinitiator in ethanol, we add the $\mathrm{ZnO}$ nanowires in a proportion of $0.5-5 \mathrm{wt} \%$, mixing the components for one hour. After waiting $24 \mathrm{~h}$ for solvent evaporation, a viscous liquid is obtained, which is used for 2PP. The microfabrication was carried out using the apparatus displayed in Fig. 1(a), with an average power of $30 \mathrm{~mW}$ (measured before the objective).

For the fs-laser micromachining, presented in Fig. 1(b), we use three motorized stages to scan the sample in $x, y$ and $z$ directions while the beam is kept still. Two different laser systems were employed, an oscillator operating at $5 \mathrm{MHz}$ with pulses of $50 \mathrm{fs}$ and $100 \mathrm{~nJ}$ (pulse energy), and an amplified system with repetition rate of $1 \mathrm{kHz}$ and pulses of $150 \mathrm{fs}$ and $400 \mu \mathrm{J}$ (pulse energy), both systems centered at $800 \mathrm{~nm}$. For the micromachining, we focused the laser beam into the sample using a microscope objective with numerical aperture (NA) of 0.65 . This parameter determines the width of the focal volume and, consequently, the feature size of the micromachined area. The samples are placed on a computercontrolled $x-y-z$ stage, which moves the sample with a constant speed, while the objective lens remains fixed, as illustrated in Fig. 1(b). The sample scan speed was $100 \mu \mathrm{m} / \mathrm{s}$ and $10 \mu \mathrm{m} / \mathrm{s}$ when using the amplified and the oscillator systems, respectively.

Glass samples containing copper or silver ions were used to obtain the respective metallic nanoparticles by fs-laser micromachining. For the copper ions we used a borosilicate glass as host matrix - $\left(50 \mathrm{SiO}_{2}-17 \mathrm{~B}_{2} \mathrm{O}_{3}-11.5 \mathrm{MgO}-10 \mathrm{Na}_{2} \mathrm{O}-11.5 \mathrm{Al}_{2} \mathrm{O}_{3}\right): 0.1 \mathrm{CuO}$ (mol\%), named $\mathrm{Cu}: \mathrm{BSi}$, while for the silver a matrix composed of $\left(42.5 \mathrm{~B}_{2} \mathrm{O}_{3}-15 \mathrm{SiO}_{2}-42.5 \mathrm{BaO}\right): \quad 0.1 \mathrm{CeO}_{2}-0.05 \mathrm{Ag}_{2} \mathrm{O}_{3} \quad$ (mol\%) was 
a

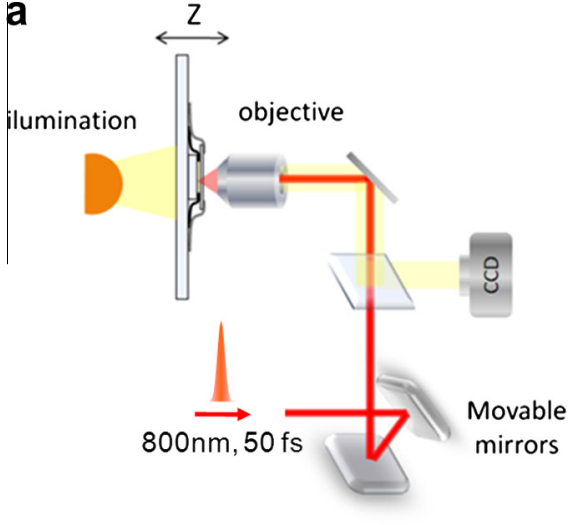

b

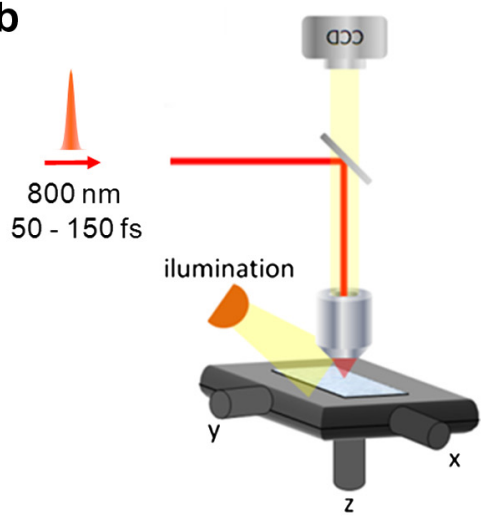

Fig. 1. Experimental setup for two-photon polymerization (2PP) (a) and fs-laser micromachining (b).

employed, named AgBBO. Both glass samples were synthesized by conventional melting-quenching technique, using high purity metal oxides and $\mathrm{Na}_{2} \mathrm{CO}_{3}, \mathrm{BaCO}_{3}, \mathrm{AgNO}_{3}$ as raw materials. The stoichiometric mixture of the reagents was melted in a platinum crucible, using an electric furnace open to the atmosphere. The liquid was quenched into a preheated stainless-steel mold and annealed to minimize the mechanical stress. For the copper doped glass the melting and annealing temperature/time were $1400^{\circ} \mathrm{C}$ for $1 \mathrm{~h}$ and $400{ }^{\circ} \mathrm{C}$ for $12 \mathrm{~h}$, respectively. For the silver doped glass, these temperatures were $1200^{\circ} \mathrm{C}$ for $2 \mathrm{~h}$ and $350^{\circ} \mathrm{C}$ for $24 \mathrm{~h}$.

The glass transition temperature $\left(T_{\mathrm{g}}\right)$ was determined by differential scanning calorimetry (DSC), using a Netzsch STA 409C equipment, in $\mathrm{Al}_{2} \mathrm{O}_{3}$ pans, within a range of $20-900{ }^{\circ} \mathrm{C}$ with a heating rate of $10^{\circ} \mathrm{C} / \mathrm{min}$, and in a synthetic air atmosphere. From the DSC curve of the $\mathrm{Cu}$ :BSi glass, we obtained $T_{\mathrm{g}}=(495 \pm 2){ }^{\circ} \mathrm{C}$ and no exothermic peak was found, indicating that there is no formation of crystalline phases during the heating up to $900^{\circ} \mathrm{C}$. The $T_{\mathrm{g}}$ of the $\mathrm{Ag}$ :BBO glass was determined as $(597 \pm 2)^{\circ} \mathrm{C}$, and the onset of the crystallization peak as $(694 \pm 2)^{\circ} \mathrm{C}$. For annealing at $650{ }^{\circ} \mathrm{C}$, no crystalline phase was observed for times smaller than $3 \mathrm{~h}$. However, for long times the $\beta$-BBO could be indexed on the X-ray diffraction pattern.

All samples obtained by two-photon polymerization and fs-laser micromachining were analyzed by optical microscopy, fluorescence microscopy, scanning electron microscopy (SEM) and UV-Vis absorption spectroscopy. The formation of metallic nanoparticles was confirmed by transmission electron microscopy (TEM).

\section{Results and discussions}

\subsection{Hybrid nanomaterials composed of gold and zinc oxide nanostructures in a polymeric matrix}

By using the indirect doping method described in Tribuzi et al. [30], we prevent the presence of gold nanoparticles during the 2PP microfabrication, which can be deleterious to the microfabrication process. SEM investigations (results not shown) evidences that the fabricated microstructures display structural integrity and smooth surface.

The microstructures doped with Au nanoparticles present a strong fluorescence, which is not observed in the non-heated $\mathrm{HAuCl}_{4}$ doped microstructures, evidencing the presence of gold nanoparticles. Fig. 2 shows a confocal fluorescence microscopy image, obtained using excitation at $450 \mathrm{~nm}$ ( $40 \times$ objective), of a microstructure containing Au nanoparticles. Such results indicate that Au nanoparticles are distributed homogeneously in the microstructure bulk, since homogenous fluorescent emission has been observed from distinct $x-y$ planes of the microstructure.

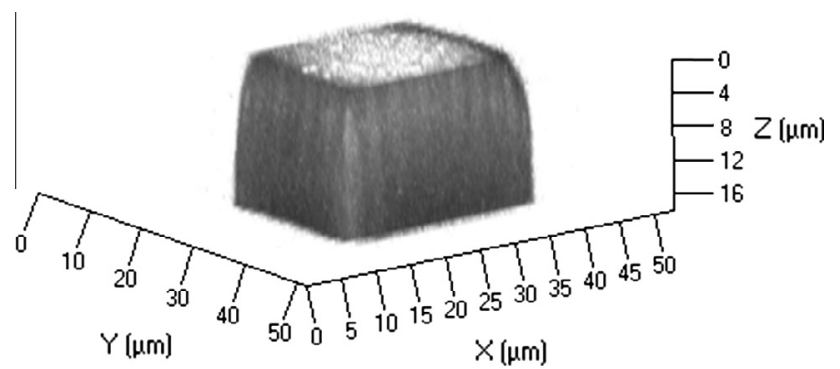

Fig. 2. Fluorescence confocal microscopy image of a microstructure containing Au nanoparticles.

Fig. 3 shows fluorescence microscopy images obtained from the emission of the microstructures under excitation with light of a wavelength of $550 \mathrm{~nm}$ (a), $475 \mathrm{~nm}$ (b) and $360 \mathrm{~nm}$ (c). As it can be seen in Fig. 3, we have been able to obtain emission from the microstructures covering a broadband of the visible spectrum ${ }^{1}$, from the red (a), up to yellow (b) ending to the blue (c). The blue emission in Fig. 3(c) probably results from the fluorescence of Au nanoparticles [30], whereas the red and green emissions (Fig. 3( $\mathrm{a}$ and $\mathrm{b})$ ) are probably due to a local field enhancement of the fluorescence of the polymeric resin, since in these cases the excitation wavelength is close to the plasmon absorption band of $\mathrm{Au}$, centered at $540 \mathrm{~nm}$. It is important to mention that no emission is observed if there are no nanoparticles into the microstructures. These results demonstrate that microstructures fabricated by $2 \mathrm{PP}$ doped with metal nanoparticles could be used in applications where high emission intensity is required such as, for instance, for micro RGB displays.

Fig. 4 shows a SEM image (tilted view) of microstructures fabricated by $2 \mathrm{PP}$ containing $\mathrm{ZnO}$ nanowires. The SEM image indicates that the presence of the $\mathrm{ZnO}$ nanowires does not affect the 2PP process, since the microstructures present good structural integrity, indicating that the approach based on a polymer matrix containing semiconductor nanostructures is well-suited for the development of hybrid devices.

Given the interesting prospects of polymeric microstructures doped with $\mathrm{ZnO}$ nanowires for optoelectronic and photonic applications, we investigated the nonlinear optical properties of the fabricated microstructures by measuring the two-photon excited emission of the doped samples. In this case, the nonlinear nature of the absorption enables spatial localization of the excitation. Besides, the use of red-shifted wavelengths as excitation source

\footnotetext{
${ }^{1}$ For interpretation of color in Fig. 3, the reader is referred to the web version of this article.
} 

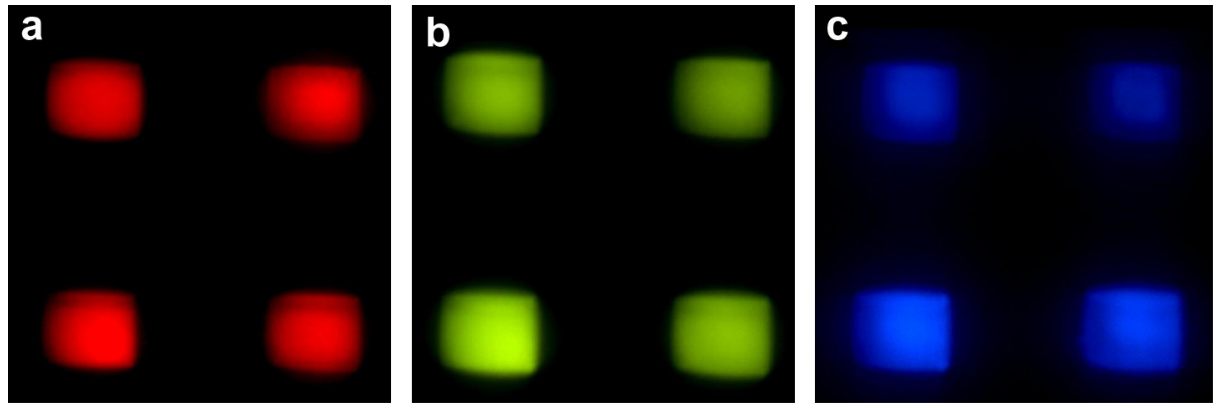

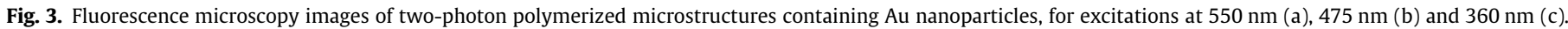

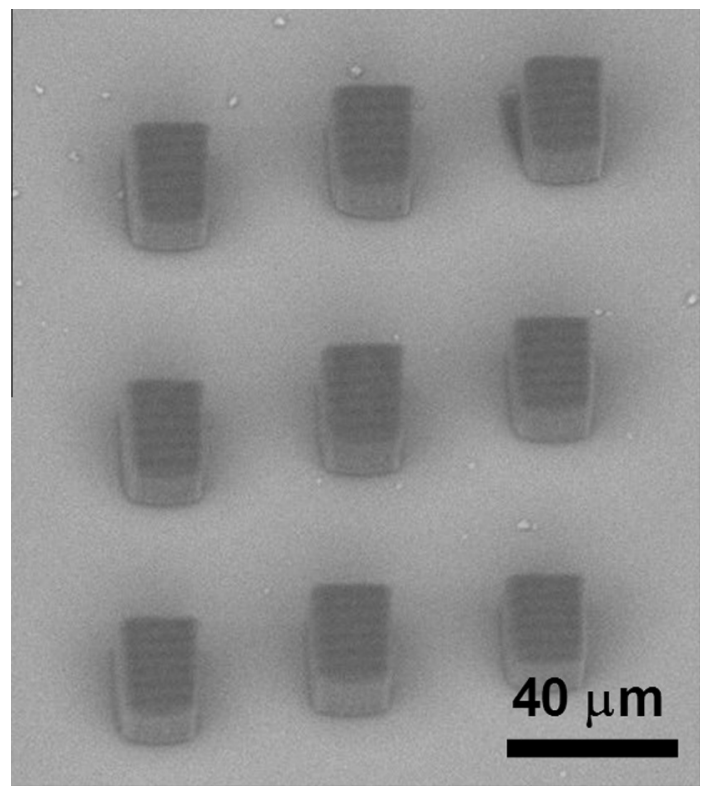

Fig. 4. SEM images of $2 \mathrm{PP}$ microstructures containing $\mathrm{ZnO}$ nanowires in a proportion of $0.5 \mathrm{wt} \%$.

decreases light scattering, increasing light penetration depth. To observe the fluorescence of the microstructures, we have set up a system that makes use of an optical microscope and a highly sensitive CCD camera (5-Megapixel). The two-photon excitation is carried out by applying $150 \mathrm{fs}$ pulses from a Ti:sapphire amplified system operating at $775 \mathrm{~nm}$ and $1 \mathrm{kHz}$. After adjusting the focus of the microstructure image on the microscope, we turn on the laser excitation and collect the fluorescence images of the microstructures. This procedure is repeated for different excitation irradiances (in the order of tenths of $\mathrm{GW} / \mathrm{cm}^{2}$ ). The results were analyzed using an image processing software, from which we obtained the average emission intensity. Fig. 5 presents, on a $\log -\log$ scale, the emission intensity (average value of image intensity) of the $\mathrm{ZnO}$ doped-microstructures as a function of excitation irradiance. The solid line in this figure represents a linear fit (in the $\log -\log$ scale) having a slope of two. This quadratic dependence of the fluorescence on the excitation intensity reveals the two-photon absorption origin of the process.

\subsection{Hybrid nanomaterials composed of copper and silver nanoparticles in glassy matrices}

\subsubsection{Copper doped borosilicate glass (Cu:BSi)}

Fig. 6(a) shows the absorption spectrum of the Cu:BSi sample before irradiation with fs-pulses (as prepared). The sample has a wide absorption band in the range of $550-1000 \mathrm{~nm}$, that is related

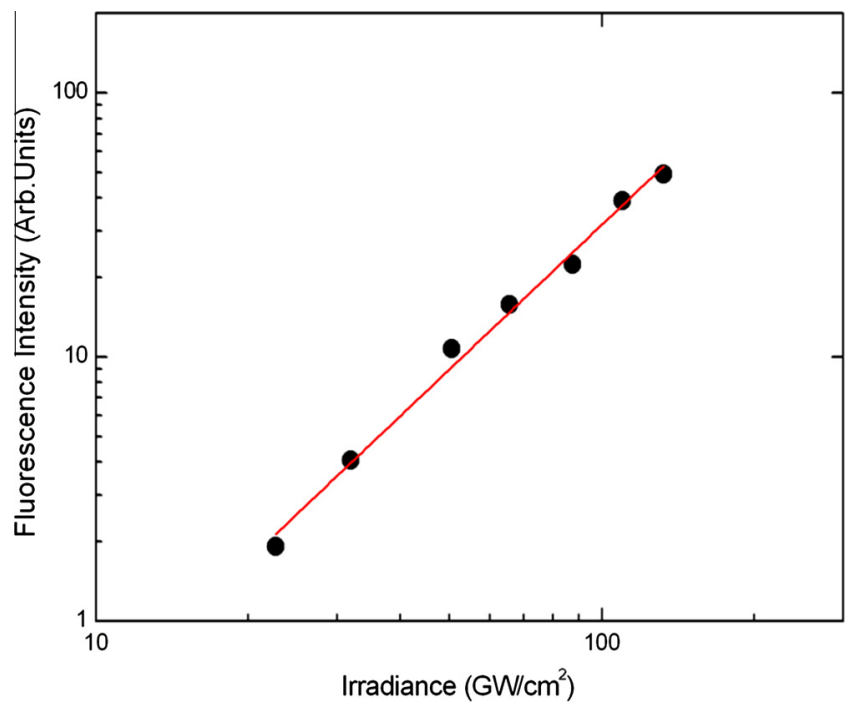

Fig. 5. Fluorescence intensity (open circles) as a function of excitation irradiance of $5 \mathrm{wt} \% \mathrm{ZnO}$ doped microstructures. The linear fit in the log-log scale (solid line) yields a slope of two, revealing the two-photon absorption origin of the process.

to $\mathrm{Cu}^{2+}$ and confers the bluish color to the sample [31]. The black line in Fig. 6(b) displays the absorption spectrum of the Cu:BSi sample after irradiation with the oscillator system (5 MHz). In this case, we used a $40 \times$ microscope objective and translated the sample perpendicularly to the incident light at a speed of $10 \mu \mathrm{m} / \mathrm{s}$, with an average laser power of $370 \mathrm{~mW}$. Under such conditions, the sample is subjected to $1.5 \times 10^{6}$ pulses/spot $(\sim 3 \mu \mathrm{m})$ and each spot experiences a fluence of $1.6 \mathrm{MJ} / \mathrm{cm}^{2}$. The irradiated area is composed of approximately 300 lines of $3 \mathrm{~mm}$ in length, separated by $15 \mu \mathrm{m}$. The absorption band observed around $570 \mathrm{~nm}$ in Fig. 6(b) corresponds to the plasmon band of copper nanoparticles $[21,32]$. It is worth mentioning that the $\mathrm{Cu}$ nanoparticles have been produced exclusively by the fs-laser irradiation, without the need of subsequent thermal treatment on the sample. When the $\mathrm{Cu}: \mathrm{BSi}$ sample is irradiated with the amplified laser system (40× microscope objective, translation speed of $100 \mu \mathrm{m} / \mathrm{s}$ and average laser power of $470 \mathrm{~mW}$ ), no evidence of the plasmon band is observed in the absorption spectrum. However, when the laser micromachined sample is subjected to a heat treatment $\left(600^{\circ} \mathrm{C}\right.$ for $\left.1 \mathrm{~h}\right)$, the irradiated area exhibits a plasmon band centered at $570 \mathrm{~nm}$, corresponding to the presence of the $\mathrm{Cu}$ nanoparticles, as can be seen by the green line of Fig. 6(c). The micromachining conditions used in this case correspond to 30 pulses/spot with each spot $(\sim 3 \mu \mathrm{m})$ subjected to a fluence of $0.17 \mathrm{MJ} / \mathrm{cm}^{2}$. As can be seen in the pictures in the insets of Fig. 6, after the production of $\mathrm{Cu}$ nanoparticles the irradiated area presents a reddish color. It is worthwhile to stress that the generation of $\mathrm{Cu}$ nanoparticles is only observed in the irradiated areas. 


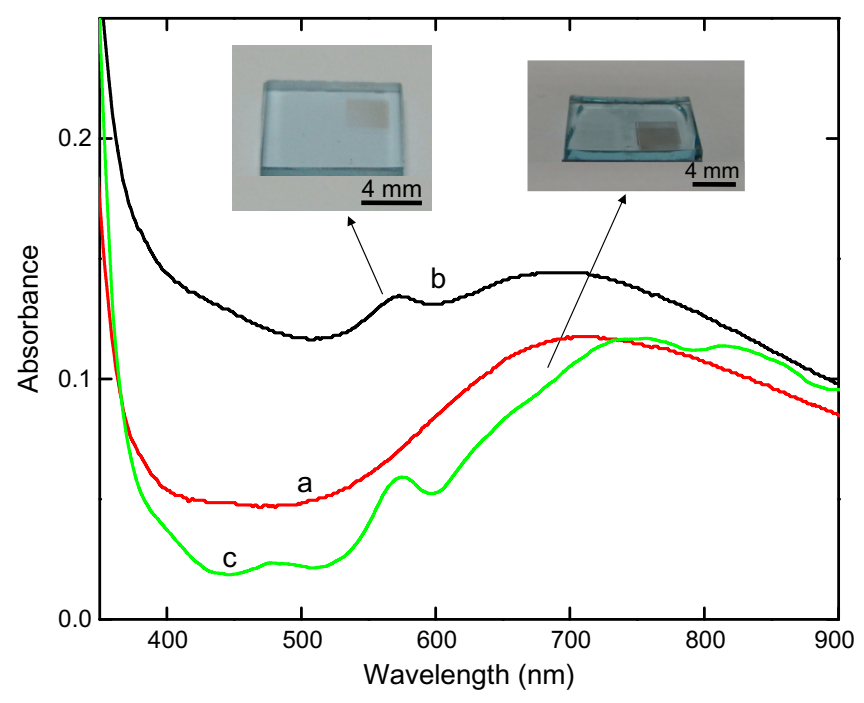

Fig. 6. Absorption spectrum of the $\mathrm{Cu}$ :BSi sample as prepared (a), after irradiation with the $5 \mathrm{MHz}$ fs-laser (b), and after irradiation with the amplified fs-laser ( $1 \mathrm{kHz})$ and subsequent thermal treatment at $600{ }^{\circ} \mathrm{C}$ for $1 \mathrm{~h}(\mathrm{c})$. The insets show pictures of the irradiated sample (b and $c$ ) after the production of the nanoparticles for each case.

To further confirm the production of $\mathrm{Cu}$ nanoparticles in the samples, we have carried out TEM measurements, whose results are presented in Fig. 7. The inset of Fig. 7 displays the electron diffraction pattern of $\mathrm{Cu}$ nanoparticles, obtained for the sample micromachined with the amplified laser system and subsequent thermal treatment. The obtained diffraction pattern reveals the formation of $\mathrm{Cu}$ nanoparticles with cubic crystal symmetry, that corresponds to group precipitates with approximately the same crystallographic orientation.

The photoreduction of $\mathrm{Cu}^{2+}$ ions, and the subsequent production of nanoparticles, is a consequence of the free electrons generated by the nonlinear light-matter interaction induced by the fs-laser excitation, which results in the formation of copper atoms $\left(\mathrm{Cu}^{0}\right)$. The nanoparticles are hence obtained upon aggregation of $\mathrm{Cu}^{0}$, which requires mobility of the neutral $\mathrm{Cu}$ atoms, attained by heating up the sample. Therefore, when high repetition

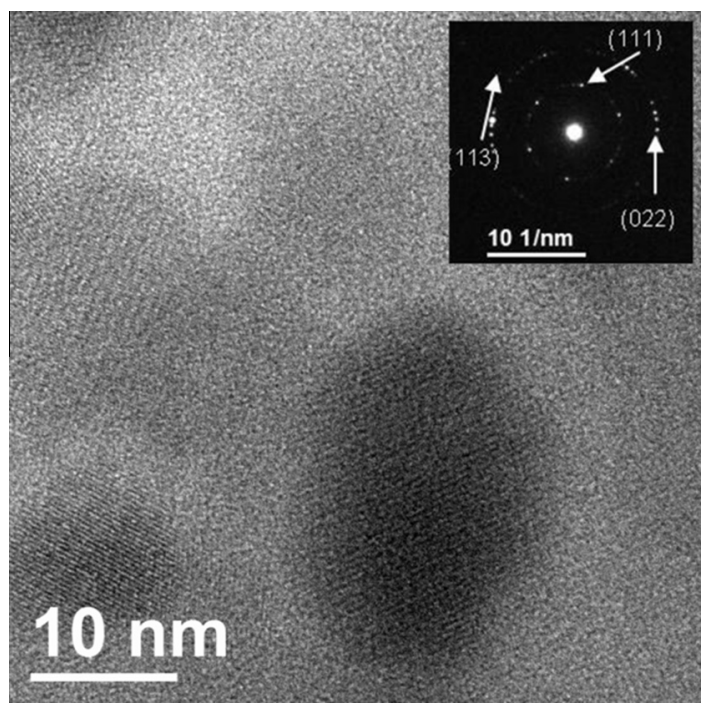

Fig. 7. TEM images obtained from the Cu:BSi sample irradiated with amplified lase system after the heat treatment. The electron diffraction pattern is shown in the inset, revealing the formation of $\mathrm{Cu}$ nanoparticles. rate laser $(5 \mathrm{MHz})$ is employed, accumulative effects originated from the sequential absorption of pulses heat the irradiated region [33] promoting diffusion and aggregation of the $\mathrm{Cu}^{0}$ atoms, leading then to the formation of nanoparticles [32-34]. On the other hand, when low repetition rates are used $(1 \mathrm{kHz})$ no thermal effects are present, because the pulse energy-matter interaction time is shorter than the time necessary to transfer the absorbed energy to the lattice [1]. For this reason, in the experiments carried out with the $1 \mathrm{kHz}$ laser system, we only observed the plasmon band upon laser irradiation and further external heat treatment, responsible for providing the atomic mobility for diffusion and formation of nanoparticles. Due to the very distinct pulse energies achieved with each laser system, the production of nanoparticles with the oscillator ( $\mathrm{MHz}$ system) requires a fluence which is one order of magnitude higher than the one employed with the amplifier laser system. It is worth mentioning that reverse saturable absorption (RSA) has been observed in copper doped glasses [35]. This process may play a positive contribution on the formation of nanoparticles, once RSA implies a higher excited-state absorption cross-section which would favor the electronic transition to the conduction band. Thus, the increase of free electron generation could improve the $\mathrm{Cu}^{2+}$ photoreduction and the formation of the metallic nanoparticles.

\subsubsection{Silver doped barium borate glass (Ag:BBO)}

In Fig. 8 we present results concerning the production of $\mathrm{Ag}$ nanoparticles in the Ag:BBO glass sample. The absorption spectrum of the as-prepared sample (non-irradiated) is shown Fig. 8(a), and displays its broad transparent window, including the spectral region at $800 \mathrm{~nm}$, where the excitation is carried out. The black line in Fig. 8(b) exhibits results obtained for the sample irradiated using the oscillator laser system ( $5 \mathrm{MHz})$, whereas the blue line (c) displays the absorption spectrum obtained for the sample using the amplified system $(1 \mathrm{kHz})$. For both laser systems the same $40 \times$ microscope objective was used (spot diameter of $\sim 3 \mu \mathrm{m}$ ). The scanning speed used for the micromaching was $10 \mu \mathrm{m} / \mathrm{s}$ for the oscillator and $100 \mu \mathrm{m} / \mathrm{s}$ for the amplified laser system. For the oscillator (Fig. 8(b)) we used an average power of $270 \mathrm{~mW}$, leading to a fluence of $1.1 \mathrm{MJ} / \mathrm{cm}^{2}$, six times higher than the amplified system $\left(0.18 \mathrm{MJ} / \mathrm{cm}^{2}\right.$, using $\left.415 \mathrm{~mW}\right)$. As shown in Fig. 8, with both systems we were able to observe the resonant

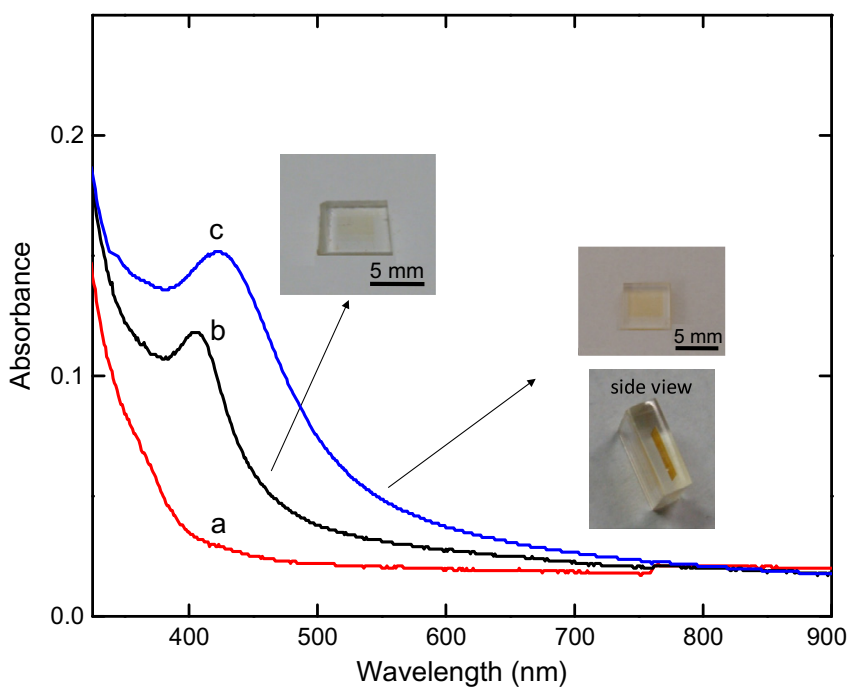

Fig. 8. Absorption spectrum of the Ag:BBO sample as prepared (a), after irradiation with the $5 \mathrm{MHz}$ fs-laser (b) and after irradiation with the amplified fs-laser (1 kHz) and subsequent thermal treatment at $400{ }^{\circ} \mathrm{C}$ for $1 \mathrm{~h}$ (c). The insets show pictures of the irradiated sample after the nanoparticles production for each case, which displays that the nanoparticles formation occurs also in the bulk (top view). 
plasmon band around $410 \mathrm{~nm}$, corresponding to the presence of the silver nanoparticles. Similarly to the Cu:BSi sample, the plasmon band for the Ag:BBO sample irradiated with $1 \mathrm{kHz}$ system was only observed after heat treatment $\left(400^{\circ} \mathrm{C}\right.$ for $\left.1 \mathrm{~h}\right)$.

The pictures in the insets of Fig. 8 shows that the production of the $\mathrm{Ag}$ nanoparticles occurs only in the fs-laser irradiated areas, conferring a yellowish color to it. It is interesting to note (see inset of Fig. 8) that the production of the Ag nanoparticles can be confined to the bulk of the sample, as a result of the nonlinear interaction that promotes the photoreduction of the Ag.

\section{Conclusions}

We employed femtosecond laser processing to produce hybrid materials, including polymeric matrices containing $\mathrm{Au}$ and $\mathrm{ZnO}$ nanostructures and borate and borosilicate glasses containing $\mathrm{Cu}$ and $\mathrm{Ag}$ nanoparticles. Specifically, we produced, by two-photon polymerization, microstructures which due to the presence of $\mathrm{Au}$ nanoparticles are fluorescent at red, green and blue colors through the excitation at 550, 475 and $360 \mathrm{~nm}$, respectively. Red and green emissions probably arise from the local field enhancement of the polymeric resin fluorescence, while the blue emission probably corresponds to the fluorescence of the Au nanoparticles. Further studies are needed to completely understand the origin of such emissions. Another hybrid material developed by two-photon polymerization was the microstructure containing $\mathrm{ZnO}$ nanowire, which displayed intense fluorescence using two-photon excitation. In addition, femtosecond laser processing was also used to macromachine boro- and borosilicate glasses to induce nucleation and growth of $\mathrm{Cu}$ and $\mathrm{Ag}$ nanoparticles in the bulk of glassy matrices, which present new plasmonic absorption bands. Both high and low repetition rate lasers can be employed to produce such nanoparticles in glasses. Nonetheless, for the production of the nanoparticles using lasers with repetition rate of $\mathrm{kHz}$ and fluence of $0.2 \mathrm{MJ} / \mathrm{cm}^{2}$ an additional heat treatment is required, while the latter in not necessary when higher fluences (6-9 times) lasers operating at $\mathrm{MHz}$ scale are employed. In summary, we demonstrated that femtosecond laser processing is a powerful tool to obtain hybrid nanomaterials, based on polymeric and glassy matrices containing metallic and semiconductor nanostructures, with interesting absorption and luminescent properties for designing optical and photonics devices.

\section{Acknowledgments}

We thank financial support from the Brazilian agencies FAPESP, CNPq and CAPES. T.V. acknowledges financial support by the German Research Foundation (DFG) through the research unit FOR1616 (project V01265/7). Authors would also like to thank
W. Avansi and the Brazilian National Synchrotron Light Laboratory (LNLS) for the TEM measurement.

\section{References}

[1] R.R. Gattass, E. Mazur, Nat. Photonics 2 (2008) 219-225.

[2] L. Kuna, C. Sommer, F. Reil, J.R. Krenn, P. Hartmann, P. Pachler, H. Hoschopf, F.P. Wenzl, Appl. Surf. Sci. 258 (2012) 9213-9217.

[3] D.S. Correa, M.R. Cardoso, V. Tribuzi, L. Misoguti, C.R. Mendonca, IEEE J. Sel. Top. Quantum Electron. 18 (2012) 176-186.

[4] A.Y. Vorobyev, C.L. Guo, Sci. Adv. Mater. 4 (2012) 432-438.

[5] K. Sugioka, Y. Cheng, Lab Chip 12 (2012) 3576-3589.

[6] S. Kawata, H.B. Sun, T. Tanaka, K. Takada, Nature 412 (2001) 697-698.

[7] S. Maruo, O. Nakamura, S. Kawata, Opt. Lett. 22 (1997) 132-134.

[8] F. Korte, J. Serbin, J. Koch, A. Egbert, C. Fallnich, A. Ostendorf, B.N. Chichkov, Appl. Phys. A-Mater. Sci. Process. 77 (2003) 229-235.

[9] H.B. Sun, S. Kawata, Two-Photon Photopolymerization and 3D Lithographic Microfabrication, NMR - 3D Analysis - Photopolymerization, Springer-Verlag Berlim, Berlin, 2004. pp. 169-273.

[10] C.N. LaFratta, J.T. Fourkas, T. Baldacchini, R.A. Farrer, Angew. Chem.-Int. Ed. 46 (2007) 6238-6258.

[11] K.S. Lee, R.H. Kim, D.Y. Yang, S.H. Park, Prog. Polym. Sci. 33 (2008) 631-681.

[12] S.R. Marder, J.L. Bredas, J.W. Perry, MRS Bull. 32 (2007) 561-565.

[13] S. Maruo, J.T. Fourkas, Laser Photonics Rev. 2 (2008) 100-111.

[14] M. Farsari, M. Vamvakaki, B.N. Chichkov, J. Opt. 12 (2010).

[15] A.M. Kowalevicz, V. Sharma, E.P. Ippen, J.G. Fujimoto, K. Minoshima, Opt. Lett. 30 (2005) 1060-1062.

[16] W. Watanabe, S. Sowa, T. Tamaki, K. Itoh, J. Nishii, Jpn. J. Appl. Phys. Part 2 Lett. Express Lett. 45 (2006) L765-L767.

[17] X. Liu, D. Du, G. Mourou, IEEE J. Quantum Electron. 33 (1997) 1706-1716.

[18] D.M. Krol, J. Non-Cryst. Solids 354 (2008) 416-424.

[19] J.R. Qiu, M. Shirai, T. Nakaya, J.H. Si, X.W. Jiang, C.S. Zhu, K. Hirao, Appl. Phys. Lett. 81 (2002) 3040-3042.

[20] J.R. Qiu, X.W. Jiang, C.S. Zhu, M. Shirai, J. Si, N. Jiang, K. Hirao, Angew. Chem.Int. Ed. 43 (2004) 2230-2234.

[21] J.M.P. Almeida, L. De Boni, W. Avansi, C. Ribeiro, E. Longo, A.C. Hernandes, C.R. Mendonca, Opt. Express 20 (2012) 15106-15113.

[22] X. Huang, S. Neretina, M.A. El-Sayed, Adv. Mater. 21 (2009) 4880-4910.

[23] Y.H. Tao, J.F. Han, C.T. Ye, T. Thomas, H.Y. Dou, J. Mater. Chem. 22 (2012) 18864-18871.

[24] J.N. Anker, W.P. Hall, O. Lyandres, N.C. Shah, J. Zhao, R.P. Van Duyne, Nat. Mater. 7 (2008) 442-453.

[25] C.J. Heo, H.C. Jeon, S.Y. Lee, S.G. Jang, S. Cho, Y. Choi, S.M. Yang, J. Mater. Chem. 22 (2012) 13903-13907.

[26] H.A. Atwater, A. Polman, Nat. Mater. 9 (2010) 205-213.

[27] A. Dev, A. Elshaer, T. Voss, IEEE J. Sel. Top. Quantum Electron. 17 (2011) 896906.

[28] T. Baldacchini, C.N. LaFratta, R.A. Farrer, M.C. Teich, B.E.A. Saleh, M.J. Naughton, J.T. Fourkas, J. Appl. Phys. 95 (2004) 6072-6076.

[29] C.R. Mendonca, D.S. Correa, T. Baldacchini, P. Tayalia, E. Mazur, Appl. Phys. AMater. Sci. Process. 90 (2008) 633-636.

[30] V. Tribuzi, D.S. Correa, W. Avansi, C. Ribeiro, E. Longo, C.R. Mendonca, Opt. Express 20 (2012) 21107-21113.

[31] N.S. Rao, L.S. Rao, Y. Gandhi, V. Ravikumar, N. Veeraiah, Phys. B - Condens. Matter 405 (2010) 4092-4100.

[32] Y. Teng, B. Qian, N. Jiang, Y. Liu, F.F. Luo, S. Ye, J.J. Zhou, B. Zhu, H.P. Zeng, J.R. Qiu, Chem. Phys. Lett. 485 (2010) 91-94.

[33] Y. Dai, G. Yu, M. He, H. Ma, X. Yan, G. Ma, Appl. Phys. B-Lasers Opt. 103 (2011) 663-667.

[34] Y. Teng, J.J. Zhou, F.F. Luo, G. Lin, J.R. Qiu, J. Non-Cryst. Solids 357 (2011) 2380 2383.

[35] D. Manzani, J.M.P. Almeida, M. Napoli, L.D. Boni, M. Nalin, C.R.M. Afonso, S.J.L. Ribeiro, C.R. Mendonça, Plasmonics (2013), http://dx.doi.org/10.1007/s11468013-9585-z. 